\title{
Preface for Hybrid Materials
}

Hybrid Materials or organic/inorganic hybrid materials originated out of a series of books and symposia with the general theme "Better Ceramics Through Chemistry," starting in the early '80s. Hybrid Materials has since blossomed into a very large and diverse field of research ranging from "Polymer Precursors to Ceramics" to "Structured Materials with Hierarchical Designs" to novel nanocomposite monoliths and thin films to porous materials that permit gas sieving, catalysis and that also offer novel electronic and photonic properties. All of these areas take advantage of combinations of organic components mixed with inorganic components.

The current issue of AOC includes selected papers from a May 2012 U.S.-Japan workshop on Hybrid Materials held at the University of Michigan, the fifth in a series spanning roughly 20 years. These papers are meant to give the reader a general overview of various efforts centered primarily in the U.S. and Japan on the development of tools for use in designing hybrid materials, some of their properties, analytical tools available to characterize such materials and some important directions in the area of applied materials science.

Richard M. Laine Professor

The University of Michigan, Materials Science and Engineering

Director

Macromolecular Science and Eng.,

2114 H.H. Dow Bldg,

Ann Arbor, Ml 48109-2136

734-764-6203, Cell 358-9996, talsdad@umich.edu

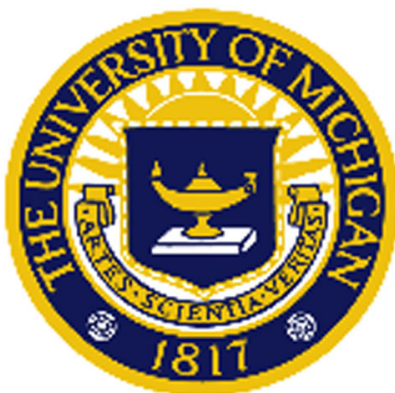

